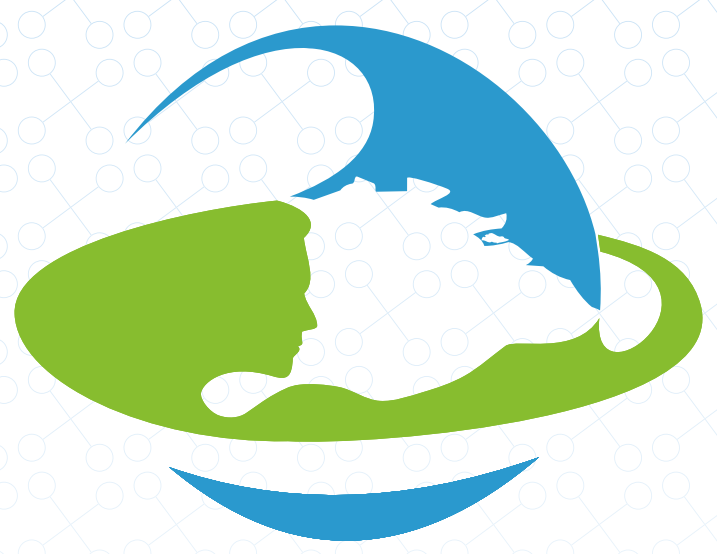

\title{
CURSO AUTOINSTRUCIONAL EM TELESSAÚDE: UMA VISÃO GERAL
}

\author{
AUTOINSTRUCIONAL COURSE IN TELEHEALTH: AN OVERVIEW
}

Alexandre Medeiros de Figueiredo

Diretor do Departamento de Educação na Saúde/SGTES/MS, Mestre em epidemiologia da UFRGS. Residência Médica em Clínica Médica no Hospital Santa Marcelina em São Paulo e de Medicina de Família e Comunidade do Serviço de Saúde Comunitária do Grupo Hospitalar Conceição em Porto Alegre, Graduação em Medicina pela UFPB, alexandre.figueiredo@saude.gov.br

Tâmara Albuquerque Leite Guedes

Mestre em Educação pela UFPB, Especialista em Política e Gestão do Cuidado, Especialista em Fisioterapia em Terapia Intensiva, Graduação em Fisioterapia pela UFPB, tamara.guedes@saude.gov.br

Thaís Maíra de Matos

Coordenação Nacional Telessaúde Brasil Redes; Departamento de Gestão da Educação na Saúde - DEGES;

Secretaria de Gestão do Trabalho e Educação na Saúde - SGTES. thais.matos@saude.gov.br

Ricardo Alexsandro de Medeiros Valentim

Coordenador do Laboratório de Inovação Tecnológica em Saúde do Hospital Universitário Onofre Lopes; Professor do Departamento de Engenharia Biomédica, da Universidade Federal do Rio Grande do Norte

(UFRN); Av. Nilo Peçanha, 620, Petrópolis - Natal/RN, Natal, Brasil. ricardo.valentim@ufrnet.br

Bruno Gomes de Araujo

Professor de Sistemas de Informação do IFRN, Doutor em Engenharia Elétrica e da Computação pela UFRN, Editor da Revista Brasileira de Inovação Tecnológica em Saúde (R-BITS), Pesquisador do Laboratório de Inovação Tecnológica em Saúde (LAIS - HUOL - UFRN), bruno.gomes@ifrn.edu.br

Custodio Leopoldino de Brito Guerra Neto

Doutorado em Ciência e Engenharia UFRN; Mestrado em Engenharia Mecânica UFRN; Especialista em

Periodontia (Academia Norte-riograndense de Odontologia do RN); Especialista em Implantodontia

(Associação Brasileira de Odontologia - ABO-RN) Graduação em Odontologia UFRN.; Professor do

Departamento de Engenharia Biomédica UFRN; Pesquisador do LAIS (Laboratório de Inovação Tecnológica em Saúde), custodioguerra@yahoo.com.br
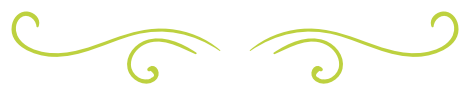

\section{RESUMO}

O Programa Nacional Telessaúde Brasil Redes tem como objetivo melhorar a qualidade do atendimento e no Sistema Único de Saúde (SUS) e ofertar estratégias de apoio assistencial que fortaleçam a integração entre os serviços de saúde. Isto é possível através dos núcleos de telessaúde e suas atividades-fim: teleconsultoria; telediagnóstico; teleducação; e segunda opinião formativa. A teleconsultoria desempenha um papel importante na qualidade da atenção, e vem sendo estimulada para que seja a principal oferta

do programa como retaguarda assistencial para a Atenção Básica. Uma estratégia para a divulgação do programa e da própria atividade, é a capacitação através da teleducação. Diante disto, foi criado um curso autoinstrucional em telessaúde para que qualquer trabalhador da saúde possa ter acesso e possa se qualificar de forma independente.

PALAVRAS-CHAVE: Telessaúde, EaD, Autoinstrucional, Teleconsuloria. 


\section{ABSTRACT}

The Programa Nacional Telessaúde Brasil Redes aims to improve the quality of care and the Sistema Único de Saúde (SUS) and offer assistance support strategies that strengthen the integration of health services. This is possible through the telehealth center and its activities: teleconsulting; remote diagnostics; distance learning; and formative second opinion. The teleconsulting perform an important role in the quality of care, and has been encouraged to be the main program's offer as care support to Primary Care. One strategy for the dissemination of the program and the activity is the training through distance learning. Given this, it created a autoinstrucional course about telehealth to any health worker may have access and can make aqualification independently.

KEYWORDS: Telehealth, Distance education, Autoinstrucional, Teleconsulting.

\section{INTRODUÇÃO}

O Programa Nacional Telessaúde Brasil Redes desempenha um papel muito importante na Educação Permanente em Saúde (EPS) das equipes de Atenção Básica (AB) localizadas em qualquer ponto do país, melhorando a qualidade do atendimento e no Sistema Único de Saúde (SUS).

Ele foi instituído em 2007, com o nome de Programa Nacional Telessaúde Brasil, pela Secretaria de Gestão do Trabalho e da Educação na Saúde (SGTES), e redefinido e ampliado em 2011 através da Portaria 2.546/2011, passando a ser denominado Programa Nacional Telessaúde Brasil Redes, objetivando também ofertar estratégias de apoio assistencial que fortaleçam a integração entre os serviços de saúde, ampliando a resolutividade desses serviços (BRASIL, 2011).

Atualmente, o Programa Nacional Telessaúde Brasil Redes está presente em todas as Unidades da Federação, podendo ter núcleos de telessaúde de abrangências estadual ou intermunicipal. Na Figura 1, é possível analisar a cobertura dos estados que possuem núcleos de telessaúde, implantados e em fase de implantação. Percebe-se que há uma grande cobertura no território nacional do telessaúde.

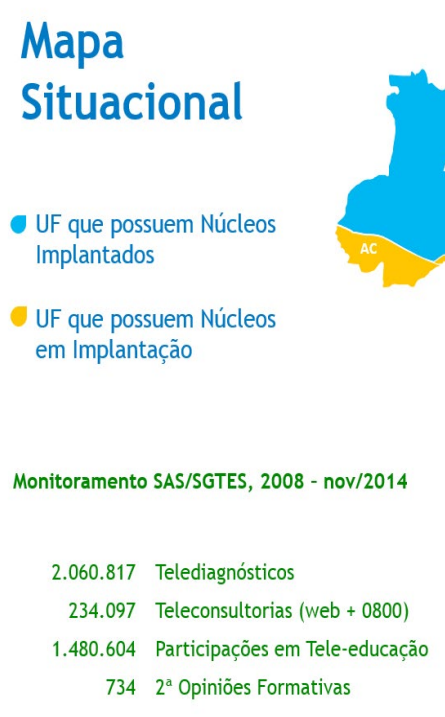

FONTE: SGTES/SAS/MS, dezembro/2014

Figura 1 - Mapa situacional dos estados com núcleos de telessaúde implantados e em fase de implantação, financiados com recursos do Ministério da Saúde (SGTES e SAS), dezembro/2014.

Fonte: Secretaria de Gestão do Trabalho e da Educação na Saúde/ Secretaria de Atenção à Saúde/ Ministério da Saúde.

As atividades-fim dos núcleos telessaúde são: teleconsultorias; telediagnósticos; teleducação; e Segunda Opinião Formativa. Essas atividades podem ser registradas em plataformas on-line, nas quais são possíveis cadastrar usuários e estabelecimentos que utilizam a ferramenta, bem como sistematizar e monitorar a produção dessas atividades.

A ampliação do Telessaúde Brasil Redes teve início pela Atenção Básica, programa que já apresentava histórico de resultados positivos. Os núcleos de telessaúde atendem ao princípio de que a Atenção Básica funciona como ordenadora da rede, sendo assim, as teleconsultorias são sempre analisadas primeiramente pelo médico de família e da comunidade ou por profissionais com experiência em Atenção Básica. Os casos são remetidos a especialistas focais apenas quando necessário. Os resultados alcançados com a implantação do Programa 
Telessaúde Brasil Redes demonstram um avanço significativo nos processos de qualificação dos profissionais de saúde, especialmente, para aqueles que atuam nos municípios de difícil acesso (BRASIL, 2012).

Um dos desafios atualmente encontrado nesta expansão é o de capacitar um maior número de trabalhadores da saúde para que possam atuar de forma correta pelo Programa, e utilizar os seus serviços. Os cursos à distância se mostram importantes ferramentas na solução deste desafio, já que existem ambientes virtuais que proporcionam fornecimento universal e instantâneo de grandes quantidades e variedades de informações em rede on-line (DA SILVA ABBAD, 2014) (DE ARAÚJO MENDONÇA e MENDONÇA, 2010).

Uma das modalidades de curso à distância é o autoinstrucional, no qual disponibiliza ao aluno um material autoexplicativo e de fácil aprendizado. Nesta modalidade, o aluno interage apenas com o computador. Desta forma, o presente artigo tem como objetivo apresentar um curso autoinstrucional em telessaúde, feito para capacitar qualquer trabalhador de saúde.

\section{VISÃO GERAL DO}

\section{TELESSAÚDE NO BRASIL E PRINCIPAIS DESAFIOS}

Atualmente existem 45 núcleos implantados e 11 núcleos em fase de implantação. Em 8 estados e no Distrito Federal, os recursos repassados pelo Ministério foram unificados para a estruturação de um único núcleo com abrangência estadual. É importante destacar que as atividades do Telessaúde vão muito além de uma "resposta a dúvidas clínicas". Alguns núcleos, ligados a universidades, têm desenvolvido pesquisas e atuado na construção de materiais de apoio institucional tanto para implantação de novos núcleos quanto para facilitar a organização do processo de trabalho de novos núcleos.

O Programa Telessaúde vem ganhando visibilidade e se tornando foco de interesse da gestão a partir das evidências apresentadas, tornando-se uma ferramenta importante de Regulação, atuando na redução de encaminhamentos para especialidades, reduzindo filas de espera por atendimento com especialistas e custos para a gestão.

Um dos serviços utilizado para tais finalidades, é o Teleconsultoria, e deve ser a principal ferramenta de interação entre os trabalhadores das unidades de saúde e teleconsultores para atuar diretamente na qualidade da atenção. Ela tem sido estimulada por todos os núcleos de telessaúde como principal oferta do programa como retaguarda assistencial para a Atenção Básica. Apesar do crescimento, ainda se faz necessário ampliar a utilização em todos os pontos de telessaúde em funcionamento. É necessário articular o debate da teleconsultoria à utilização dos protocolos clínicos junto às equipes de saúde da família, apoiando a política de regulação.

Junto com a teleducação são potenciais na reestruturação do processo de trabalho da equipe de saúde. As ações em teleducação também têm sido estimuladas para que sejam baseadas nas necessidades dos trabalhadores no cotidiano das práticas, fortalecendo a política de Educação Permanente em Saúde.

Além disso, de maio a setembro de 2014, foi realizada uma pesquisa nacional de avaliação do programa Telessaúde, realizada pela Ouvidoria do Ministério da Saúde. O objetivo foi de verificar a implantação e utilização do Programa Telessaúde Brasil Redes e ainda divulgar informações para quem não o conhece, visando contribuir para sua melhoria.

A pesquisa foi realizada por meio de inquérito telefônico operacionalizado pelos atendentes do Disque Saúde 136, para um público alvo de 119.108 profissionais de saúde de 19.906 Unidades Básicas de Saúde. Foram entrevistados 17.365 profissionais de saúde, destes 8.408 relataram conhecer o Telessaúde (48,4\%). Dentre os profissionais que conhecem o programa os médicos e enfermeiros são os profissionais que mais conhecem. Contudo, nos questionamentos sobre a utilização, verificou-se que dos profissionais que conhecem o programa apenas $40 \%$ o utilizam no seu dia a dia.

É notório que o Programa sente a necessidade de métodos que ajudem a divulgar 
suas ações, como também capacitar um maior número de profissionais. A teleducação pode ajudar através dos seus vários métodos de ensino à distância. Um deles vem ganhando destaque na área da saúde, que é o curso autoinstrucional.

Apesar da falta de conexão com a internet ser um problema relatado por núcleos de telessaúde, o Plano Nacional de Banda Larga é a estratégia apontada para superar esse desafio. As unidades de saúde da família que fazem parte do PMAQ serão contempladas com internet de banda larga. Com esse processo em andamento espera-se que com o avanço da conectividade tenhamos um número potencial de unidades de saúde conectadas e com possibilidade de fazer parte do Telessaúde.

\section{CURSO AUTOINSTRUCIONAL EM TELESSAÚDE}

O curso autoinstrucional é um modelo de ensino à distância que vem se popularizando nos últimos anos. Ele se diferencia dos demais por garantir a autonomia e independência do aluno através de um material autoexplicativo (RAMOS, 2010). Ele deve expor o conteúdo de maneira objetiva e simples, de forma que o aluno saiba conduzir o curso.

Um curso autoinstrucional deve permitir que os alunos possam se matricular de forma independente e a qualquer momento, ficando livre para cursar o material disponível de acordo com a sua disponibilidade de tempo.

Diante disto, o curso autoinstrucional em Telessaúde foi criado, já que um dos objetivos do Programa é abranger um maior número de trabalhadores da saúde em localidades distantes e remotas, evitando gastos com deslocamentos de equipes ou mesmo de um especialista para ministrar este curso.

O curso disponibiliza de forma acessível uma unidade com uma visão geral sobre - Programa Nacional Telessaúde Brasil Redes, e também outras ensinando a utilizar o serviço de Teleconsultoria, formando Teleconsultores e Telerreguladores para atuarem nos mais diversos Núcleos pelo Brasil. Os trabalhadores da saúde podem acessar o site do ambiente virtual de aprendizagem, se cadastrarem de forma independente, e ter acesso aos materiais do curso. A Figura 2 contém a Tela inicial do ambiente.

\section{Telessaúde: teleconsultoria e telerregulação (telessaude)}
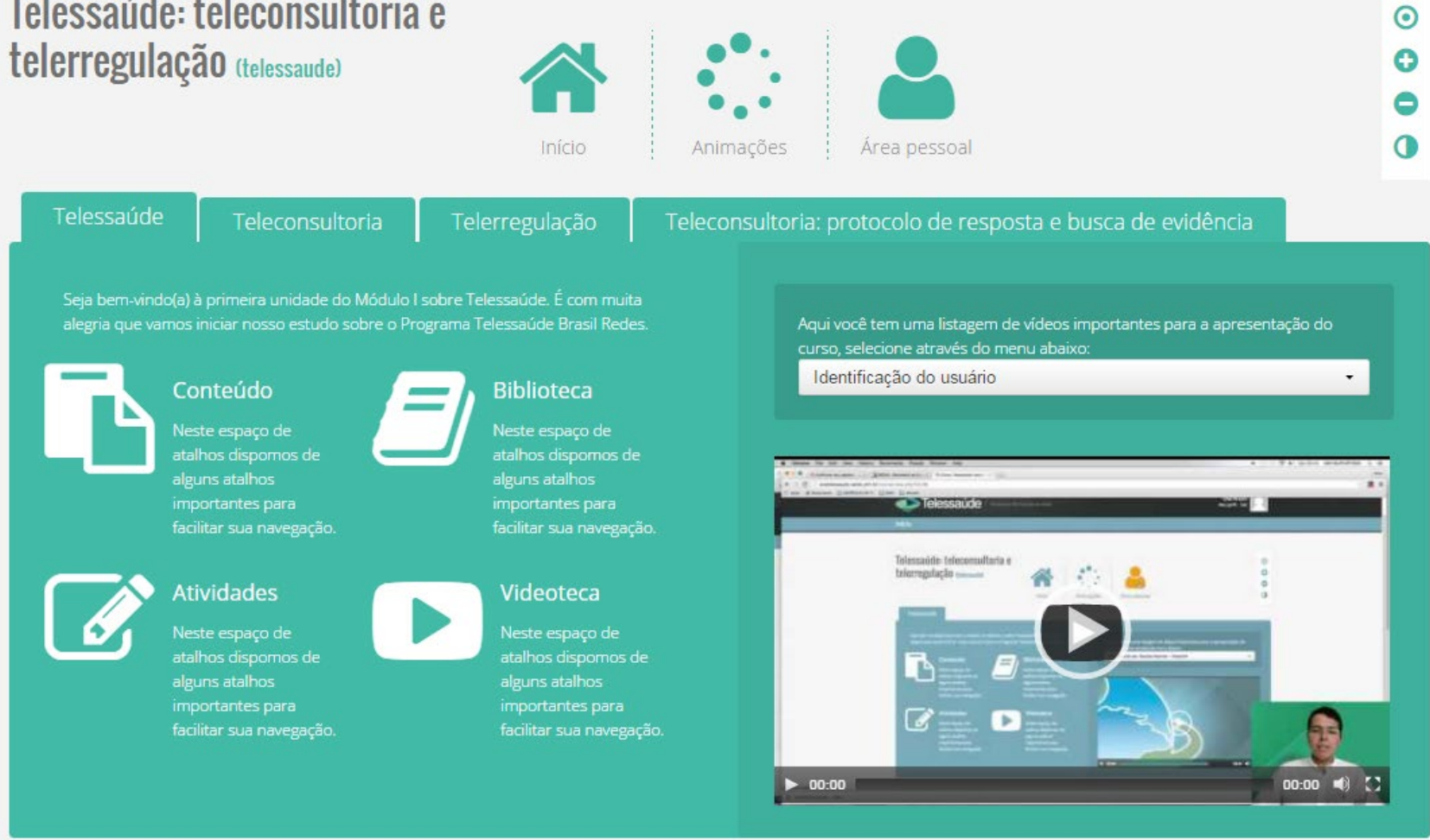

Figura 2 - Tela inicial do curso autoinstrucional. 
Além de apresentar um visual intuitivo, o ambiente disponibiliza diversos vídeos que ensinam como utilizar cada item do curso, localizados na tela inicial. É uma forma de ajuda para os alunos que tem menos familiaridade com a própria informática.

O ambiente apresenta de forma clara quais unidades o curso tem (Telessaúde,
Teleconsultoria, Telerregulação e Protocolo de Resposta e busca de evidência) em abas que são exibidas durante todo o curso. Ao entrar em uma determinada unidade, o aluno tem acesso ao Conteúdo, Atividades, Biblioteca e aos vídeos na Videoteca. A Figura 3 representa a navegação pelo conteúdo da Unidade 1 do curso.

\section{Telessaúde

\section{Telessaúde: Princípios e diretrizes}

\section{Página anterior (1) 2 (3) 4 Próxima página}

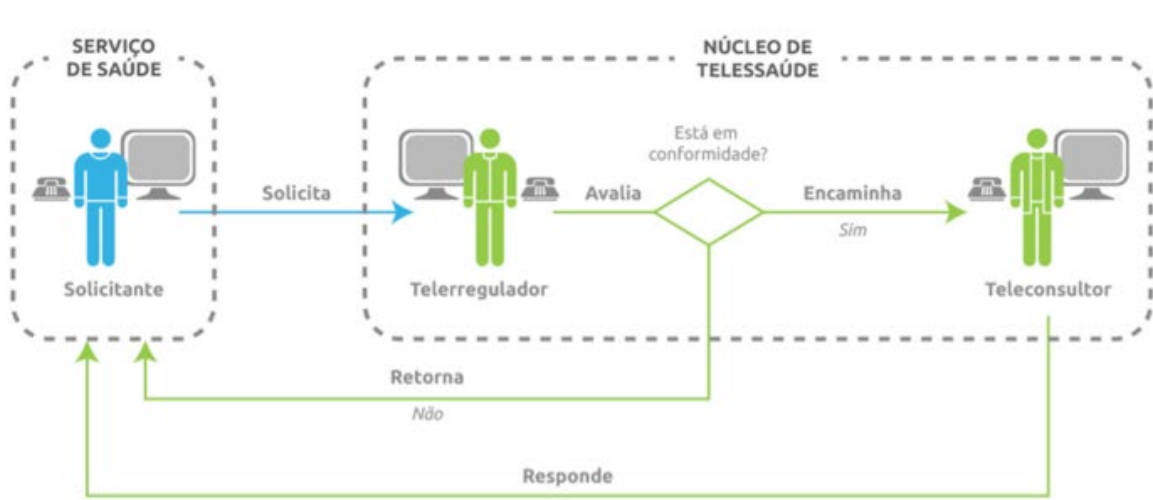

Figura 1 - Representação do serviço de Teleconsultoria

Fonte: llustração de Roberto Lima (2015).

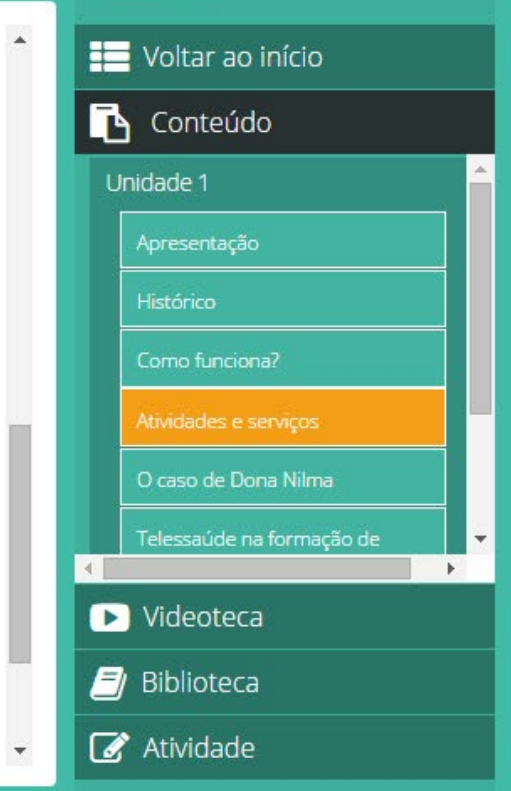

Figura 3 - Exibição de Conteúdos no Ambiente.

Ainda na Figura 3, é possível visualizar uma imagem representando o serviço de teleconsultoria. $\bigcirc$ conteúdo multimídia foi criado especificamente para este curso, seguindo um padrão de cores, simplicidade e explicação, proporcionando um rápido entendimento e aprendizado.

$\mathrm{Na}$ Figura 4, existe uma animação que auxilia o entendimento de qual é o papel do telerregulador no processo de solicitação de uma nova teleconsultoria. Neste exemplo, o aluno interagi definindo se a teleconsultoria se adequa ou não ao padrão aceito (clicando em sim ou não). Dependendo da escolha, a animação mostra caminhos diferentes para a teleconsultoria. 


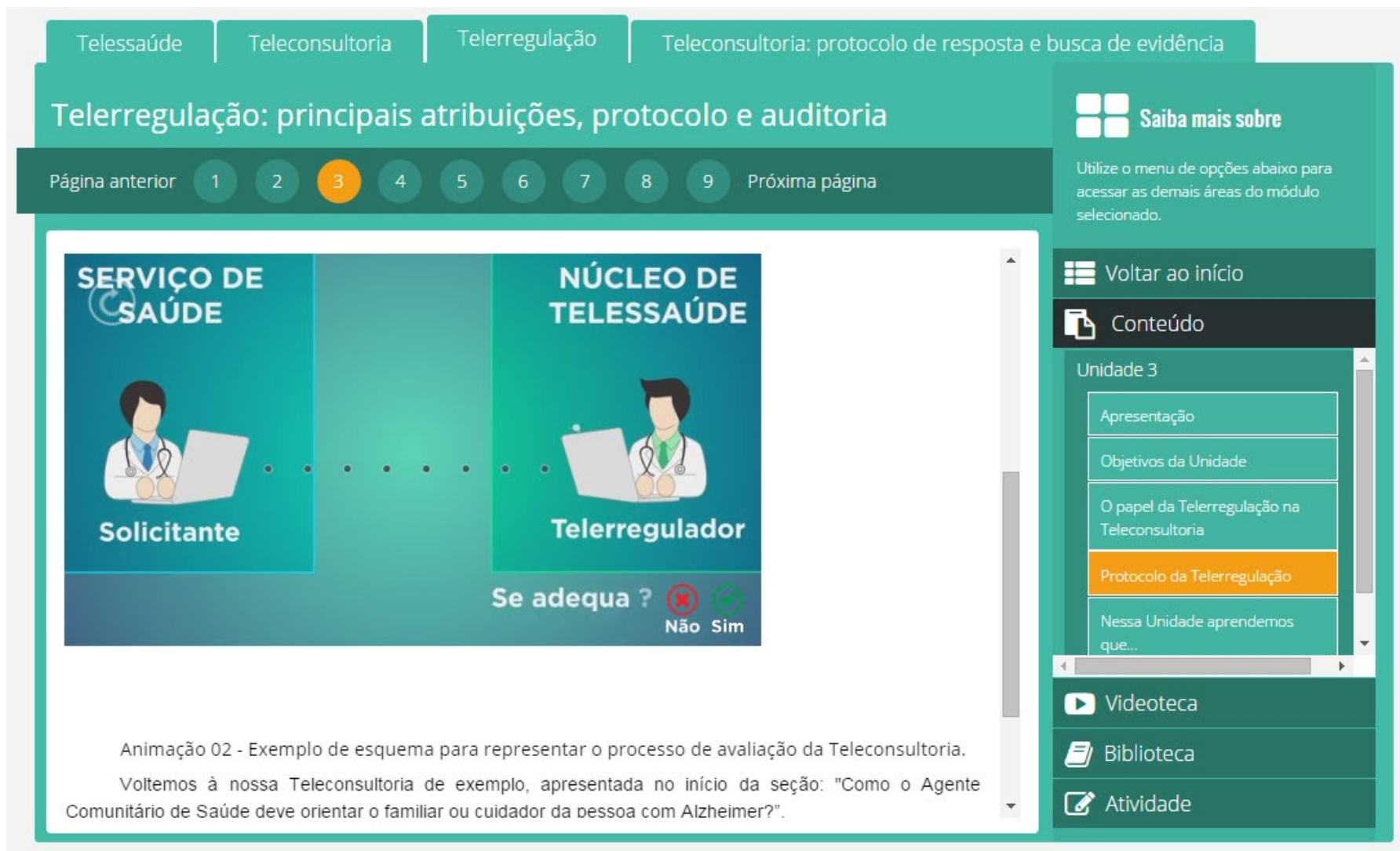

Figura 4 - Exemplo de animação.

Ao final de cada unidade, o usuário deverá responder às atividades. São elencadas de forma aleatória 5 questões, e o usuário deverá acertar $60 \%$ para que possa continuar no curso e acessar a próxima unidade. A Figura 5 contém duas questões referentes a unidade 1 do curso.

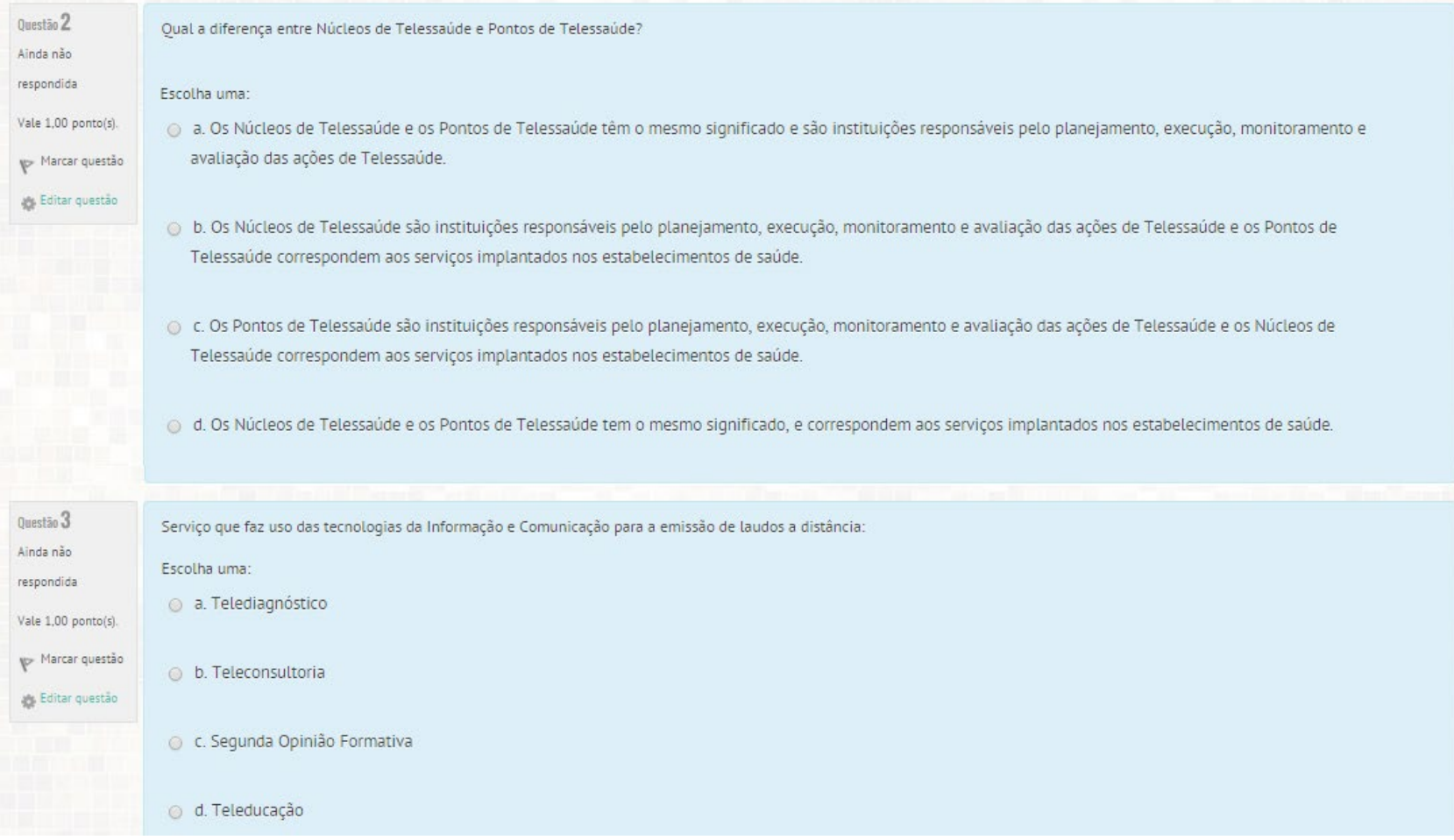

Figura 5 - Atividades do curso. 
Para facilitar e preparar o aluno para as atividades, outra estratégia utilizada no curso foi dar destaque a determinados trechos do material (textos em negrito, cor de fundo e realce) que estão relacionados às questões. Desta forma, indiretamente o aluno está se preparando para responder a avaliação final de cada unidade, e fixar as informações mais importantes do material.

\section{CONSIDERAÇÕES FINAIS}

Diante das questões apresentadas, é notório o crescimento do Telessaúde desde sua criação em 2007. Desde a quantidade de pontos implantados, crescimento na utilização de serviços até sua complexa rede de núcleos. Espera-se que a superação dos desafios apontados contribua para o fortalecimento e novas perspectivas para o programa. Uma vez que os resultados do programa mostram benefício para trabalhadores e população.

Como um dos objetivos do Programa é o de capacitar um maior número de trabaIhadores da saúde, a criação de um curso autoinstrucional se torna uma importante estratégia para este fim. Desta forma, qualquer trabalhador tem acesso ao ambiente virtual de forma independente e pode acompanhar o curso no momento que achar propício.

O curso autoinstrucional em telessaúde permite que os trabalhadores de saúde adquiram maiores conhecimentos sobre o Programa, sejam capacitados para utilizarem o serviço de teleconsuloria, e possam atuar como teleconsultores e telerreguladores. Tudo isso através de um material didático e interativo que foi criado para facilitar o aprendizado dos alunos. 


\section{REFERÊNCIAS}

BRASIL. Ministério da Saúde. Custeio dos Núcleos de Telessaúde MANUAL INSTRUTIVO / Ministério da Saúde. Brasília: Ministério da Saúde, 2015. p.: il. (Série A. Normas e Manuais Técnicos).

BRASIL. Ministério da Saúde. Manual de Telessaúde para

Atenção Básica / Atenção Primária à Saúde / Ministério da Saúde, Universidade Federal do Rio Grande do Sul. - Brasília: Ministério da Saúde, 2012. 123 p. : il. - (Série A. Normas e Manuais Técnicos).

BRASIL. Ministério da Saúde. Portaria n².546, de 27 de outubro de 2011 que Redefine e amplia o Programa Telessaúde Brasil, que passa a ser denominado Programa Nacional Telessaúde Brasil Redes (Telessaúde Brasil Redes). 2011.

Disponível em: <http://bvsms.saude.gov.br/bvs/saudelegis/ gm/2011/prt2546_27_10_2011.html >. Acesso em 20 jul. 2015.

DA SILVA ABBAD, Gardênia. Educação a distância: o estado da arte e o futuro necessário. Revista do Serviço Público, v. 58, n. 3, p. 351-374, 2014.

DE ARAÚJO MENDONÇA, Gilda Aquino; MENDONÇA, Alzino Furtado de. UTILIZAÇÃO DE AMBIENTES VIRTUAIS NO APOIO AO APRENDIZ NA EAD. 2010. Disponível em: <http://www.abed. org.br/congresso2010/cd/1942010094738.pdf>. Acesso em: 5 jan. 2016.

RAMOS, Daniela Karine. Aspectos pedagógicos e tecnológicos da concepção e desenvolvimento de propostas de E-learning. Colabor@-A Revista Digital da CVA-RICESU, v. 3, n. 9, 2010. 